\title{
Japan's rocketing ambitions
}

\section{Tokyo}

WITH the successful launch of its lunar probe (see below), Japan's academic space agency ISAS is considering its future plans and, in particular, whether there is a need for increased cooperation with foreign agencies or its domestic big brother, the National Space Development Agency (NASDA), to aim at more ambitious missions.

In the immediate future, the situation is unlikely to change with scientific missions beyond the Earth still the prerogative of ISAS while NASDA concentrates its efforts on the launch of Earth-orbiting applications satellites. But later on, the two agencies may have to join forces.

ISAS's present plans are for the launch of a radiotelescope, using the new $\mathrm{M}-\mathrm{V}$ rocket, into a $20,000-\mathrm{km}$ eccentric Earth orbit in 1994 or 1995 to form a very-long baseline interferometry network with radiotelescopes on Earth. And in the next few months Jun Nishimura, director general of ISAS, says the institute will choose one of the three other missions being considered for 1996: a lunar spacecraft that will drop penetrometers containing seismographs to monitor moonquakes, release of a balloon into the atmosphere of Venus, and a mission to collect dust from the comet Wirtanen.

But the $\mathrm{M}-\mathrm{V}$ rocket will only be able to send small scientific payloads to the planets and Minoru Oda, former director general of ISAS, sees it partly as carrying out "scouting missions" that could be followed by bigger projects involving international collaboration or joint projects with NASDA using its much more powerful $\mathrm{H}-2$ rocket. But both Oda and Nishimura are anxious to retain ISAS's independent capability to carry out small scientific missions at low cost.

ISAS has several joint missions with the United States coming up in the next few years (see below) and collaboration might next be extended to the Soviet Union. Nishimura says that in December he had very preliminary discussions with Albert Galeev, director of the Space Research Institute in Moscow, about joining the Soviet Union and other nations in a mission to Mars in 1994 or later.

Tomifumi Godai, executive director of NASDA, agrees that ISAS and NASDA may cooperate in the launch of large scientific payloads. But NASDA has its own plans for sending remote-sensing satellites to the Moon or planets and is even considering a manned lunar base.

For at least the next decade NASDA will be fully occupied with Earth-orbiting missions, the largest being the US space station. The Japanese Experiment Module (JEM) for the station now scheduled for launch in early 1998 will cost about
$¥ 300,000$ million (\$2000 million) to develop and will have annual operating costs of about $¥ 40,000$ million, according to Tada-aki Mochida of NASDA who presented a cost analysis for JEM at a workshop last month in Tokyo.

NASDA and STA have long hoped to separate the budget for JEM from NASDA's general budget so that the space station does not become a millstone around NASDA's neck. But the Ministry of Finance has resisted such requests, says Yasushi Horikawa, operations manager for NASDA's space station office. Unless there is a dramatic increase in NASDA's budget (which is unlikely) JEM will swallow about a third of NASDA's annual budget.

NASDA is hoping to recover some of JEM's costs by allowing Japanese private

\section{Moon next stop}

\section{Uchinoura}

JAPAN last week became the third nation to launch a spacecraft to the Moon. But besides the giant lunar programmes of the Soviet Union and the United States, Japan's efforts cost next to nothing.

Japan's moonshot is the work of the Institute of Space and Astronautical Science (ISAS), a small academic space agency affiliated with the Ministry of Education, Science and Culture. On Wednesday 24 January, one of the institute's M-3SII solid fuel rockets successfully carried a tiny $182-\mathrm{kg}$ satellite into an elliptical orbit around the Earth in a night launch from the ISAS space centre at Uchinoura in southern Kyushu.

The satellite, named Hiten after a Buddhist angel, will orbit the Earth four-and-a-half times before being pulled by gravity towards the approaching Moon on 18 March. As the satellite swings by the Moon at an altitude of about $18,000 \mathrm{~km}$, it will release a mini-satellite about the size of a basket-ball into lunar orbit.

The mini lunar orbiter will transmit information back to Earth on its temperature and position for 30 days. Meanwhile the mother satellite, after orbiting the Earth another three-and-half-times, will swing by the Moon again at an altitude of about 35,000 km on 10 July.

Hiroki Matsuo of ISAS who is in charge of the mission says the main purpose is not to reach the Moon but rather to test the swing-by technique that will be used in future lunar and planetary missions, such as the GEOTAIL mission, a joint project with the United States scheduled for 1992 (see 'Working with NASA'). The only scientific instrument aboard Hiten is a Munich Dust Counter which will determine the speed and size of particles that hit the satellite as it ventures up to distances of 700,000 km from Earth.

The total cost of Japan's first moonshot including the M-3SII rocket is about $\$ 50$ million dollars. companies to carry out experiments in JEM. But the re-phasing of the space station by the United States last year has caused a loss of confidence and Japanese companies will probably wait and see what happens before committing themselves.

NASDA sees the station as a way of developing an independant capability for manned space flight. Oda and Nishimura, on the other hand, are less enthusiastic about manned missions. Oda's personal opinion has long been that there should be greater use of robots and automation on the space station - something which may well come about because of the US decision last year to reduce the number of crew members from 8 to 4 during the first few years of operation. But if Japan is going to send men into space, "we should use them" Oda says. Nishimura says that ISAS will probably carry out some projects on the space station.

David Swinbanks

\section{Working with NASA}

\section{Tokyo}

JAPAN and the United States have signed an agreement to launch a Japanese spacecraft into the tail of the Earth's magnetic field. The GEOTAIL mission is another example of the growing collaboration between the two countries in space.

The GEOTAIL satellite will be built by Japan's ISAS space agency and is scheduled to be launched in July 1992 by the US National Aeronautics and Space Administration (NASA) on a US Delta II rocket.

The spacecraft will examine the Earth's magnetic field on the nightside of the planet where it is drawn out into a long tail by the solar wind. The aim of the mission is to clarify energy transport and conversion in the magnetosphere, in particular the interactions between the solar wind and the magnetic field.

The satellite will perform a double swingby of the Moon, taking measurements in a region extending from 8 to 220 times the Earth's radius before moving into an elliptical orbit closer to the planet, in the equatorial magnetosphere.

GEOTAIL is one of several joint USJapanese space projects proposed for the next few years. At the end of last month, the Japanese cabinet approved an initial budget for a project to monitor tropical rainfall with a US satellite to be launched on a Japanese H-2 rocket in the mid-1990s. Next year, ISAS will launch an X-ray satellite, SOLAR $A$, to monitor solar flares with US and Japanese X-ray telescopes. In 1995, Japan's other space organization, the National Space Development Agency (NASDA), will launch its Advanced Earth Observation Satellite (ADEOS) which will monitor the Earth's climate and ozone layer using sensors developed by NASA, NASDA and the European Space Agency. And, of course, Japan is a major participant in the US space station project.

Davld Swinbanks 